\title{
Spatial entanglement and massive neutrino oscillations produced by orbital electron capture decay
}

\author{
I. M. Pavlichenkov 1, * \\ ${ }^{1}$ Russian Research Center Kurchatov Institute, Moscow, 123182, Russia
}

(Dated: June 20, 2018)

\begin{abstract}
The two-particle wave function of neutrino and recoil nucleus is found as a solution of an initial value problem in the far zone for a time longer than the electron capture decay lifetime of a hydrogenlike ion. The neutrino-recoil entanglement arising in such a process is a consequence of the momentum conservation and is closely related to the wave packet structure of the state. Because of neutrino mixing, the joint wave packet involves the coherent superposition of the neutrino mass eigenstate packets. This is the new physical realization of the Einstein-Podolsky-Rosen thought experiment, which has no analogue in quantum optics and quantum informatics. A class of possible experiments for the registration of a neutrino and a recoil nucleus is proposed. It is shown that due to spatial correlations neutrino oscillations can be observed in the coincidence experiment with the recoil.

PACS numbers: 14.60.Pq, 23.40.-s, 03.65.Ud
\end{abstract}

\section{INTRODUCTION}

Massive neutrino oscillations are a consequence of the presence of flavor neutrino mixing and a clear evidence of physics beyond the standard model. They are subject of an intense experimental and theoretical research beginning with the pioneering paper by Pontecorvo [1]. The theoretical approach is mostly phenomenological and uses the plane wave [2 4 ] or the wave packet formalism [5 -9] to describe the evolution of the massive neutrino states, and the field-theoretical approach [10 12 which takes into account the processes of production, propagation and detection of neutrino. In spite of the fact that almost all these treatments provide the canonical formula for the probability of oscillations, some basic issues of the theory of neutrino oscillations are still being debated 13]. In particular, there is no consensus on whether the three neutrino mass eigenstates have equal energies or equal momenta. Furthermore, the wave packet formalism leaves unanswered the question of how the properties of a neutrino wave packet are determined by the process of weak decay.

If we want to resolve these problems, we need to do away with ad-hoc assumptions and invoke basic principles of quantum mechanics to describe the weak decay of an unstable object and neutrino production [14]. Closely related problems have been studied in quantum optics. Fedorov et al. [15, 16] considered the decay of composite objects into two fragments that are free to move away from the breakup point and are constrained only by momentum and energy conservation. The authors obtained position dependent two particle wave function as the solution of an initial-value problem. This quantum state is entangled and closely related to the Einstein-PodolskyRosen one [17].

*Electronic address: pavi@mbslab.kiae.ru
Our motivation for this work is to apply the formalism of Refs. [15, 16] to the description of orbital electron capture (EC) decay. In this scenario, the spontaneous emission of a flavor neutrino by an atom is described in three dimensions with initial wave function of a decaying atom taken in the form of a finite-size wave packet. The recoil-neutrino wave function is found analytically in the coordinate representation as the solution of the timedependent Schrödinger equation in a far zone. Its wave packet structure evolves with time. The function does not factorize in the neutrino, $\mathbf{r}_{\nu}$, and recoil, $\mathbf{r}_{n}$, spatial coordinates, which is a direct indication that the quantum state of the system is entangled. However, due to neutrino mixing the entanglement involves the coherent superposition of mass eigenstates with equal energies and different momenta that manifests itself in the oscillating correlations of the two particles.

Entanglement means that knowledge of one of the particles reveals information about the other. Space correlations of neutrino and recoil nucleus are the experimental implication of this feature. It should be emphasized that the spatial entanglement which we are considering, has nothing to do with kinematic entanglement (see, for example, Refs. [4, 18]) which does not involve an exact solution of the Schrödinger equation. Spatial entanglement between an atom and a photon in spontaneous emission has been observed by Kurtsiefer et al. 19].

Because of strong spatial correlations, we are able to observe with two detectors neutrino oscillations and a recoil simultaneously in the coincidence measurement. This experiment, first considered by Dolgov et al. [20], could be of interest for the next generation of neutrino detectors. By contrast, registration of one particle independent of the other disentangles the recoil-neutrino pair. For example, to observe neutrino oscillations the recoil position is ignored whereas a flavor neutrino is detected. This scenario is used currently to observe neutrino oscillations in reactor and accelerator experiments.

The paper is organized as follows. In Sec. II we ap- 
ply the Weisskopf-Wigner theory of spontaneous emission 21] to treat the EC-decay process of the ion confined to a small volume in a trap. We specifically study a hydrogenlike (H-like) ion of the intermediate mass region with a $\mathrm{K}$-electron in the lowest hyperfine state having the total angular momentum $F=I \pm 1 / 2$, where $I$ is the nuclear spin. Such ions have been used in the much-talked-of GSI experiment [22] and its second run in 2010 [23]. However, the question of what happens to the neutrino and the recoil nucleus on time scales comparable to the lifetime of the parent ion is left unanswered. The main result of this section is the recoil-neutrino wave function that describes the spatiotemporal behavior of these particles. In Sec. III we use the far zone approximations to express this function as a product of the relative and the center of mass (CM) wave functions. It is shown that the coherent superposition of the neutrino mass eigenstates has a fixed kinetic energy equal to the EC-decay energy. The structure and time evolution of the relative motion (RM) and the CM wave packets are investigated in Sec. IV. The far zone formalism allows to find the spreading of these wave packets. In Sec. V we outline the experiments appropriate for the detection of a flavor neutrino and a recoil nucleus. Our findings are summarized in Sec. VI. The Appendix contains the details of calculation of an integral from Sec. III.

\section{ORBITAL ELECTRON CAPTURE DECAY OF MOVING HYDROGEN-LIKE ION}

The decay which we study is the capture of a single electron from the K-shell into a completely ionized daughter nucleus and a electron neutrino, as final state. The system is described by the Hamiltonian $H=H_{0}+V_{s f}+H_{w}$, where the unperturbed Hamiltonian has the form

$H_{0}=\frac{\hat{\mathbf{p}}^{2}}{2 M}+H_{n}\left(\boldsymbol{\rho}_{1}, \ldots, \boldsymbol{\rho}_{A}\right)+\sum_{\lambda} \varepsilon_{\lambda} a_{\lambda}^{+} a_{\lambda}+\sum_{i, \mathbf{k}} \epsilon_{i}(k) c_{i \mathbf{k}}^{+} c_{i \mathbf{k}}$.

Here $\hat{\mathbf{p}}$ is the nucleus momentum operator; $H_{n}$ is the nuclear hamiltonian depending on nucleon coordinates, $\boldsymbol{\rho}_{k}=\mathbf{r}_{k}-\mathbf{r}_{n}$, with respect to $\mathbf{r}_{n}$ which to a good approximation is the nucleus CM; $A$ is the mass number; $a_{\lambda}^{+}$ and $a_{\lambda}$ are the creation and the annihilation operators of a bound electron in the state with the energy $\varepsilon_{\lambda}$ and the quantum numbers $\lambda=n, j, m ; V_{s f}$ is a hyperfine interaction; and $c_{i \mathbf{k}}^{+}$and $c_{i \mathbf{k}}$ are the creation and the annihilation operators of the three massive neutrinos $(i=1,2,3)$ with momentum $\mathbf{k}$, the energy $\epsilon_{i}=\sqrt{k^{2}+m_{i}^{2}}$ and the mass $m_{i}$. The mass $M$ of parent and daughter particles is supposed to be equal with an accuracy of the small parameter $Q_{E C} / M$, where $Q_{E C}$ is the decay energy.

The weak-interaction Hamiltonian density is given by

$$
h_{w}(\mathbf{r})=\frac{G_{F}}{\sqrt{2}} V_{u d}[\mathbf{j}(\mathbf{r}) \cdot \mathbf{J}(\mathbf{r})+\text { H.c. }],
$$

where the lepton current,

$$
\mathbf{j}(\mathbf{r})=i \bar{\nu}_{e}(\mathbf{r}) \gamma\left(1+\gamma_{5}\right) e(\mathbf{r}),
$$

involves the flavor neutrino and electron field operators. The former is 24]

$$
\nu_{e}(\mathbf{r})=\sum_{i} U_{e i} \nu_{e}(\mathbf{r})=\sum_{i, \mathbf{k}_{i}} U_{e i} c_{i \mathbf{k}_{i}} u_{L}\left(\mathbf{n}_{i}\right) e^{i \mathbf{k}_{i} \mathbf{r}},
$$

where $U$ is the Pontecorvo-Maki-Nakagava-Sakata mixing matrix. The Dirac spinor for left-handed neutrino,

$$
u_{L}(\mathbf{n})=\frac{1}{\sqrt{2}}\left(\begin{array}{c}
\omega_{L} \\
-\omega_{L}
\end{array}\right), \omega_{L}(\mathbf{n})=\left(\begin{array}{c}
-e^{-i \varphi / 2} \sin (\theta / 2) \\
e^{i \varphi / 2} \cos (\theta / 2)
\end{array}\right),
$$

depends on the unit vector $\mathbf{n}=\mathbf{k} / k$. We will use nonrelativistic limit for the electron operator

$$
e(\mathbf{r})=\sum_{\lambda} a_{\lambda} \psi_{\lambda}(\mathbf{r}), \quad \psi_{\lambda}=\left(\begin{array}{c}
w_{\lambda} \\
0
\end{array}\right) .
$$

For the K-electron, we have $w_{1 \frac{1}{2} m}=f_{1 s}(r) \eta_{\frac{1}{2}, m}$, where $f$ is radial and $\eta$ is spin wave functions. The nucleon current for pure Gamow-Teller transitions in a nonrelativistic approximation has the form

$$
\mathbf{J}(\mathbf{r})=g_{A} \sum_{s=1}^{A} \boldsymbol{\sigma}(s) \tau_{-}(s) \delta\left(\mathbf{r}-\mathbf{r}_{s}\right)=g_{A} \sum_{s=1}^{A} \mathbf{J}(s) \delta\left(\mathbf{r}-\mathbf{r}_{s}\right),
$$

where $\sigma$ and $\tau$ are the spin and the isospin Pauli matrices.

To find the wave packet structure of an entangled state, one needs to use the coordinate representation for the wave functions of neutrino and recoil nucleus. This implies that the Hamiltonian $H_{w}$ has to commute with the total momentum of a bipartite state in the $\mathbf{r}$ representation. By using Eqs. (21), (3) and (7) we get after integration over nuclear volume the weak interaction Hamiltonian fulfilling the momentum conservation law

$$
\begin{gathered}
H_{w}=i \frac{G_{F}}{\sqrt{2}} V_{u d} \sum_{i, \mathbf{k}_{i}} U_{e i}^{*} c_{i \mathbf{k}_{i}}^{+} \bar{u}_{L}(\mathbf{n}) \gamma\left(1+\gamma_{5}\right) \\
\times \sum_{s} \mathbf{J}(s) e^{-i \mathbf{k}_{i} \boldsymbol{\rho}_{s}} \sum_{\lambda} a_{\lambda} \psi_{\lambda}\left(\boldsymbol{\rho}_{s}\right) e^{i \mathbf{k}_{i}\left(\mathbf{r}_{\nu}-\mathbf{r}_{n}\right)}+\text { H.c.. }
\end{gathered}
$$

Now let us use the time-dependent perturbation theory to determine the temporal evolution of a decaying state for the Gamow-Teller transition $F I M_{F} \rightarrow I^{\prime} M^{\prime}$ with $I^{\prime}=I \pm 1$. The parent ion is prepared in a polarized state with the angular momentum projection $M_{F}$ along the quantized axis $Z$ of the laboratory frame $S$. The solution of the Schrödinger equation with the Hamiltonian $H$ is sought by using the following ansatz for the wave function

$$
\begin{gathered}
\Psi(t)=\sum_{\mathbf{p}} \mathcal{A}(\mathbf{p}, t)\left|a F I M_{F} ; \mathbf{p}, t\right\rangle e^{-i \mathcal{E}_{a} t} \\
+\sum_{i, M^{\prime}, \mathbf{p}, \mathbf{k}_{i}} \mathcal{B}_{i M^{\prime}}\left(\mathbf{p}, \mathbf{k}_{i}, t\right)\left|b I^{\prime} M^{\prime} ; \mathbf{p}-\mathbf{k}_{i}, \mathbf{k}_{i}, t\right\rangle e^{-i \mathcal{E}_{b i} t}
\end{gathered}
$$


where

$$
\begin{gathered}
\left|a F I M_{F} ; \mathbf{p}\right\rangle=\sum_{m, M} C_{\frac{1}{2} m, I M}^{F M_{F}} \Omega_{a I M}(\boldsymbol{\rho}) a_{1 \frac{1}{2} m}^{+}|0\rangle e^{i \mathbf{p} \mathbf{r}_{n}} \\
\left|b I^{\prime} M^{\prime} ; \mathbf{p}-\mathbf{k}_{i}, \mathbf{k}_{i}\right\rangle=\Omega_{b I^{\prime} M^{\prime}}(\boldsymbol{\rho}) c_{i \mathbf{k}_{i}}^{+}|0\rangle e^{i\left(\mathbf{p}-\mathbf{k}_{i}\right) \mathbf{r}_{n}+i \mathbf{k}_{i} \mathbf{r}_{\nu}}
\end{gathered}
$$

are the eigenvectors of $H_{0}+V_{s f}$ with the eigenvalues

$$
\mathcal{E}_{a}=E_{a I}+\varepsilon_{0}+\frac{\mathbf{p}^{2}}{2 M}, \quad \mathcal{E}_{b i}=E_{b I^{\prime}}+\epsilon_{i}+\frac{\left(\mathbf{p}-\mathbf{k}_{i}\right)^{2}}{2 M} .
$$

In Eq. (10) $\Omega(\boldsymbol{\rho})=\Omega\left(\boldsymbol{\rho}_{1}, \ldots, \boldsymbol{\rho}_{A}\right)$ and $E$ are eigenfunctions and eigenvalues of the initial $(a I)$ and final $\left(b I^{\prime}\right)$ states of nucleus, $\varepsilon_{0}$ is the energy of the ion ground state, $|0\rangle$ is the lepton vacuum, and $C_{1 / 2 m, I M}^{F M_{F}}$ are ClebschGordan coefficients. In the subsequent text we will assume that $E_{b I^{\prime}}=0$.

The differential equations for the coefficients $\mathcal{A}$ and $\mathcal{B}$ are

$$
\begin{aligned}
& i \dot{\mathcal{A}}(\mathbf{p}, t)=\sum_{i, \mathbf{k}_{i}, M^{\prime}} \mathcal{B}_{i M^{\prime}}\left(\mathbf{p}, \mathbf{k}_{i}, t\right) W_{I^{\prime} M^{\prime}}\left(\mathbf{n}_{i}\right) U_{e i} e^{i\left(\mathcal{E}_{a}-\mathcal{E}_{b i}\right) t}, \\
& i \dot{\mathcal{B}}_{i M^{\prime}}\left(\mathbf{p}, \mathbf{k}_{i}, t\right)=\mathcal{A}(\mathbf{p}, t) W_{I^{\prime} M^{\prime}}^{*}\left(\mathbf{n}_{i}\right) U_{e i}^{*} e^{-i\left(\mathcal{E}_{a}-\mathcal{E}_{b i}\right) t},(12)
\end{aligned}
$$

where the matrix element of the Hamiltonian $H_{w}$,

$$
W_{I^{\prime} M^{\prime}}\left(\mathbf{n}_{i}\right)=\frac{G_{F} V_{u d}}{\sqrt{F+1 / 2}}\left\langle f_{1 s}\right\rangle g_{A} \mathcal{M}\left(a I, b I^{\prime}\right) \xi_{I^{\prime} M^{\prime}}^{*}\left(\mathbf{n}_{i}\right),
$$

includes the radial wave function of the bound electron averaged over nuclear volume, $\left\langle f_{1 s}\right\rangle$, and the nuclear reduced matrix element $\mathcal{M}$. The spinor

$$
\xi_{I^{\prime} M^{\prime}}(\mathbf{n})=C_{I^{\prime} M^{\prime}, \frac{1}{2} \mu}^{F M_{F}} D_{\mu,-\frac{1}{2}}^{1 / 2}(\varphi, \theta, 0),
$$

where $D$ is the Wigner function and $\mu=M_{F}-M^{\prime}= \pm \frac{1}{2}$, is normalized by the condition $\sum_{M_{F}, M^{\prime}}\left|\xi_{I^{\prime} M^{\prime}}\right|^{2}=F+1 / 2$.

Suppose that a parent ion after production is stored in a trap and its motion is localized. Then we switch off the field of the trap and free spreading of the ion CM wave packet begins. If the production and stored time is far less than the life-time of the parent ion, we can measure time from the beginning of free spreading. The initial state is described by the first term of Eq. (9) for $t=0$. To be specific, suppose that the CM part of this function has in the momentum representation the Gaussian form

$$
\mathcal{A}(\mathbf{p}, t=0)=(2 \sqrt{\pi} d)^{3 / 2} \exp \left(-\frac{1}{2} d^{2} p^{2}\right) .
$$

The Eqs. (12) are easily solved in the Weisskopf-Wigner approximation with the initial conditions $\mathcal{A}(\mathbf{p}, t=0)=$ $\mathcal{A}_{0}(\mathbf{p})$ and $\mathcal{B}_{i M^{\prime}}(\mathbf{p}, \mathbf{k}, t=0)=0$. The solutions for $t \gg$ $1 / Q_{E C}$ are

$$
\mathcal{A}(\mathbf{p}, t)=\mathcal{A}_{0}(\mathbf{p}) \exp (-\Gamma t)
$$

$$
\mathcal{B}_{i M^{\prime}}(\mathbf{p}, \mathbf{k}, t)=\frac{\mathcal{A}_{0}(\mathbf{p}) W_{I^{\prime} M^{\prime}}^{*}\left(\mathbf{n}_{i}\right) U_{e i}^{*}}{\mathcal{E}_{b i}-\mathcal{E}_{a}+i \Gamma}\left[1-e^{i\left(\mathcal{E}_{b i}-\mathcal{E}_{a}\right) t-\Gamma t}\right],
$$

where

$$
\begin{aligned}
& \Gamma=\pi \sum_{i, M^{\prime}, \mathbf{k}_{i}}\left|W_{I^{\prime} M^{\prime}}\left(\mathbf{n}_{i}\right)\right|^{2}\left|U_{e i}\right|^{2} \delta\left(\mathcal{E}_{b i}-\mathcal{E}_{a}\right) \\
& =\frac{\left(G_{F} V_{u d}\right)^{2}}{2 \pi(2 F+1)}\left[\left\langle f_{1 s}\right\rangle g_{A} \mathcal{M}\left(a I, b I^{\prime}\right) Q_{E C}\right]^{2}
\end{aligned}
$$

is one half of the rate of the Gamow-Teller transition $F I M_{F} \rightarrow I^{\prime} M^{\prime}, I^{\prime}=I \pm 1$. The final expression we have derived for this value ignores a small dependence of $\Gamma$ on the parent ion velocity. Furthermore, the two small dimensionless parameters

$$
\alpha=\frac{Q_{E C}}{M}, \quad \delta_{i}=\frac{m_{i}}{Q_{E C}}
$$

allow to reduce $\Gamma$ to a conventional value for the ion at rest. The last parameter is compatible with the limit of ultra relativistic neutrinos 25].

First of all, we observe that the decay probability $P(t)$ of the parent ion is not dependent on the shape of the initial CM wave packet $\mathcal{A}_{0}(\mathbf{p})$ because of

$$
P(t)=\exp (-2 \Gamma t) \sum_{\mathbf{p}} \mathcal{A}_{0}^{2}(\mathbf{p})=\exp (-2 \Gamma t) .
$$

However the probability of emission of the electron neutrino with wave vector $\mathbf{k}$ and energy $\epsilon$ is little affected by Doppler shift

$$
P_{e}(\mathbf{k})=\sum_{M^{\prime}}\left|W_{I^{\prime} M^{\prime}}(\mathbf{n})\right|^{2} \sum_{\mathbf{p}} \frac{\mathcal{A}_{0}^{2}(\mathbf{p})}{\left(\epsilon-Q_{E C}+\frac{\mathbf{k}^{2}}{2 M}-\frac{\mathbf{p} \cdot \mathbf{k}}{M}\right)^{2}+\Gamma^{2}} .
$$

With $\mathcal{B}_{i M^{\prime}}$ taken from Eq. (16), the recoil-neutrino wave function at times $t>1 / \Gamma$ has the form

$$
\begin{aligned}
& \Psi_{I^{\prime} M^{\prime}}\left(\mathbf{r}_{n}, \mathbf{r}_{\nu}, t\right)=\sum_{i, \mathbf{p}, \mathbf{k}_{i}} \frac{\mathcal{A}_{0}(\mathbf{p}) W_{I^{\prime} M^{\prime}}^{*}\left(\mathbf{n}_{i}\right)}{\epsilon_{i}-Q_{E C}+\frac{\mathbf{k}_{i}^{2}}{2 M}-\frac{\mathbf{p} \mathbf{k}_{i}}{M}+i \Gamma} \\
& \times \exp \left\{i\left(\mathbf{p}-\mathbf{k}_{i}\right) \mathbf{r}_{n}+i \mathbf{k}_{i} \mathbf{r}_{\nu}-i \mathcal{E}_{b i} t\right\} U_{e i}^{*} c_{i \mathbf{k}_{i}}^{+}|0\rangle .
\end{aligned}
$$

It carries information on the neutrino production process and involves the coherent superposition of the electron neutrino mass eigenstate components.

\section{RECOIL-NEUTRINO WAVE FUNCTION}

Now, by using the far zone approximation and small parameters (18), we write the function (21) in the form suitable for the analysis of the wave packet structure and entanglement of the recoil-neutrino state. To begin with, let us rewrite Eq. (21) as

$$
\Psi_{I^{\prime} M^{\prime}}=\sum_{\mathbf{p}} \mathcal{A}_{0}(\mathbf{p}) \exp \left(i \mathbf{p r}_{n}-i \frac{\mathbf{p}^{2} t}{2 M}\right) \sum_{i} \chi_{i}(\mathbf{r}, t) U_{e i}^{*} c_{i \mathbf{k}_{i}}^{+}|0\rangle,
$$


where the function

$$
\chi_{i}=\sum_{\mathbf{k}_{i}} \frac{W_{I^{\prime} M^{\prime}}^{*}\left(\mathbf{n}_{i}\right) \exp \left[i \mathbf{k}_{i} \mathbf{r}-i\left(\epsilon_{i}+\frac{\mathbf{k}_{i}^{2}}{2 M}-\frac{\mathbf{p k}}{M}\right) t\right]}{\epsilon_{i}-Q_{E C}+\frac{\mathbf{k}_{i}^{2}}{2 M}-\frac{\mathbf{p} \mathbf{k}_{i}}{M}+i \Gamma}
$$

depends on the relative coordinate $\mathbf{r}=\mathbf{r}_{\nu}-\mathbf{r}_{n}$. In the laboratory frame $S$, the vector $\mathbf{r}$ is specified by polar angles $\theta$ and $\varphi$. The direction of $\mathbf{k}_{i}$ is given by angles $\vartheta_{i}$ and $\phi_{i}$.

First we transform the summation over $\mathbf{k}_{i}$ in Eq. (23) to integration. To perform integration over $d \Omega_{\mathbf{k}_{i}}$, we rotate the laboratory system $S$ with the origin $O$ in the decay point by the angles $\theta, \varphi$ to align axis $Z$ along the vector $\mathbf{r}$. In the new frame $S^{\prime}$, the vectors $\mathbf{k}_{i}$ and $\mathbf{p}$ are determined by angles $\vartheta_{i}^{\prime}, \phi_{i}^{\prime}$ and $\vartheta_{p}, \phi_{p}$, respectively. The polar angles of these vectors in the systems $S$ and $S^{\prime}$ are connected with each other by well-known formulas [26]. Owing to $k_{i} r \sim Q_{E C} t>Q_{E C} / \Gamma \gg 1$, the integrant involves rapidly oscillating function $\exp \left(i k_{i} r \cos \vartheta_{i}^{\prime}\right)$ and a slowly varying preexponential function $\mathcal{F}$ of the angle $\vartheta_{i}^{\prime}$. Integration by parts over this angle yields

$$
\begin{gathered}
\int_{0}^{\pi} \mathcal{F}\left(\vartheta_{i}^{\prime}\right) \exp \left(i k_{i} r \cos \vartheta_{i}^{\prime}\right) \sin \vartheta_{i}^{\prime} d \vartheta_{i}^{\prime} \\
=\frac{i}{k_{i} r}\left[\mathcal{F}(\pi) e^{-i k_{i} r}-\mathcal{F}(0) e^{i k_{i} r}\right]+\mathcal{O}\left(1 /\left(k_{i} r\right)^{2}\right) .
\end{gathered}
$$

Here the two terms in brackets represent incoming and outgoing spherical waves. The incoming wave in the far zone gives an exponentially small contribution for $t>1 / \Gamma$ which can be neglected in comparison with the outgoing one. The latter corresponds to $\vartheta_{i}^{\prime}=0$, and it immediately follows that $\vartheta_{i}=\theta$ and $\phi_{i}=\varphi$ in the integrant of (23). Thus, we find that $\mathbf{k}_{i} \| \mathbf{r}$ and $\mathbf{n}_{i}=\mathbf{n}=\mathbf{r} / r$ in the far zone. After integration over $d \phi_{i}^{\prime}$, one obtains

$$
\chi_{i}=\frac{W_{I^{\prime} M^{\prime}}^{*}(\mathbf{n})}{i(2 \pi)^{2} r} \int_{0}^{\infty} \frac{\exp \left[i k_{i} r-i\left(\epsilon_{i}+\frac{k_{i}^{2}}{2 M}-\frac{\mathbf{p k}_{i}}{M}\right) t\right]}{\epsilon_{i}-Q_{E C}+\frac{k_{i}^{2}}{2 M}-\frac{\mathbf{p k}}{M}+i \Gamma} k_{i} d k_{i},
$$

where $\mathbf{p k}_{i}=p k_{i} \cos \vartheta_{p}$.

To calculate the integral, we change variable $k_{i}$ by $\epsilon_{i}$ and expand the former around $Q_{E C}$

$$
k_{i}\left(\epsilon_{i}\right)=k_{i 0}+\frac{1}{v_{i}}\left(\epsilon_{i}-Q_{E C}\right)-\frac{m_{i}^{2}}{2 v_{i}^{3} Q_{E C}^{3}}\left(\epsilon_{i}-Q_{E C}\right)^{2},
$$

because main contribution to the pole in (25) comes from $\epsilon_{i}=Q_{E C}-i \Gamma$. We have retained a quadratic term to describe the spreading of the RM wave packet. Here $k_{i 0}=\sqrt{Q_{E C}^{2}-m_{i}^{2}}$ and $v_{i}=k_{i 0} / Q_{E C}$ is the group velocity of neutrino. Then the integral over $\epsilon_{i}$ can be evaluated by the residue method. The details of calculations are given in the Appendix. Upon using Eqs. (13) and (17), we find in the lowest order in the parameters $\alpha$ and $\delta_{i}$

$$
\chi_{i}=-\psi_{i}(\mathbf{n}, r, t) \exp (i \alpha \mathbf{p r})
$$

where the RM wave function of $i$ th massive neutrino is

$$
\begin{aligned}
& \psi_{i}(\mathbf{n}, r, t)=\sqrt{\frac{\Gamma}{4 \pi}} \frac{1}{r}\left\{1-\operatorname{Erf}\left[\sqrt{\frac{i}{2}}\left(\Delta_{i}+i \frac{v_{i} t-r}{\Delta_{i} D_{i}}\right)\right]\right\} \\
& \times \xi_{I^{\prime} M^{\prime}}(\mathbf{n}) \exp \left[i\left(k_{i 0} r-Q_{E C} t\right)-\left(v_{i} t-r\right) / D_{i}\right] .
\end{aligned}
$$

Here Erf is the error function, $D_{i}=v_{i} / \Gamma$ is the initial width of the RM wave packet, and

$$
\Delta_{i}=\frac{1}{D_{i}}\left(\frac{t}{M}+\frac{\delta_{i}^{3} r}{m_{i} v_{i}}\right)^{1 / 2}
$$

is a dimensionless parameter describing its spreading.

Now, integration over $d \mathbf{p}$ can be readily performed after substituting expressions (15) and (27) into Eq. (22)

$$
\begin{gathered}
\sum_{\mathbf{p}} \mathcal{A}_{0}(\mathbf{p}) \exp \left[i \mathbf{p}\left(\mathbf{r}_{n}+\alpha \mathbf{r}\right)-i \frac{\mathbf{p}^{2} t}{2 M}\right]= \\
=\frac{1}{\pi^{3 / 4}\left(d+\frac{i t}{M d}\right)^{3 / 2}} \exp \left[-\frac{\left(\mathbf{r}_{n}+\alpha \mathbf{r}\right)^{2}}{2 d\left(d+\frac{i t}{M d}\right)}\right] .
\end{gathered}
$$

In view of the definition of the $\mathrm{CM}$ vector for a relativistic system in terms of the particle energies [28],

$$
\mathbf{R}=\mathbf{r}_{n}+\frac{\epsilon_{i}}{\epsilon_{n}+\epsilon_{i}} \mathbf{r} \approx \mathbf{r}_{n}+\alpha \mathbf{r},
$$

we find an analytic expression for the wave function (22)

$$
\begin{aligned}
\Psi_{I^{\prime} M^{\prime}}(\mathbf{R}, \mathbf{r}, t)= & -\frac{1}{\pi^{3 / 4}\left(d+\frac{i t}{M d}\right)^{3 / 2}} \exp \left[-\frac{\mathbf{R}^{2}}{2 d\left(d+\frac{i t}{M d}\right)}\right] \\
& \times \sum_{i} \psi_{i}(\mathbf{n}, r, t) U_{e i}^{*} c_{i \mathbf{k} 0}^{+}|0\rangle .
\end{aligned}
$$

The two-particle wave function (32) describes the evolution of the recoil-neutrino state after decay at time $t>1 / \Gamma$. This expression is accurate to within the small parameters $\alpha$ and $\delta_{i}$ (18). The function carries information on the decay process and has the form of a product of the CM and RM parts. Such a factorization of the total wave function is a general feature of the two-particle decay of noninteracting fragments constrained only by momentum and energy conservation [16]. The RM wave function involves the coherent superposition of the mass eigenstate components of the electron neutrino state.

The time-dependent phase factor of the recoil-neutrino wave function (32) [see also Eq. (28)] involves the kinetic energy of the pair. As shown in the Appendix, it is equal with great accuracy to the decay energy $Q_{E C}$. Thus, all three massive neutrinos have the same energy. This resolves the long standing problem "same energy or same momentum." This issue, like other paradoxes of neutrino oscillations [13], emerges from the theory that considers the time evolution of a neutrino independently from a recoil. The exact solution (32) shows that a neutrino and a recoil do not evolve separately due to their spatial correlation. 


\section{THE WAVE PACKET STRUCTURE OF THE RECOIL-NEUTRINO STATE AND SPATIAL ENTANGLEMENT}

The spatiotemporal behavior of the joint quantum state of the recoil and the electron neutrino following the EC-decay is in agreement with the results obtained in the theoretical studies of decaying bipartite systems [15, 16, 27]. The distinctive feature of our system is the coherent superposition of neutrino mass eigenstates. In this section we give the more detailed analysis of the function (32) providing insight into the nature of entanglement and neutrino oscillations. We begin with the wave packet structure of this function, since entanglement and neutrino oscillations depend on spatial localization of particles involved in the decay.

The CM part of $\Psi_{I^{\prime} M^{\prime}}(\mathbf{R}, \mathbf{r}, t)$ has the form of a spreading wave packet

$$
\left|\Psi_{C M}(\mathbf{R}, t)\right|^{2}=\frac{1}{\pi^{3 / 2} D_{R}^{3}(t)} \exp \left[-\frac{\mathbf{R}^{2}}{D_{R}^{2}(t)}\right],
$$

with the time-dependent width

$$
D_{R}(t)=\left[d^{2}+\left(\frac{t}{M d}\right)^{2}\right]^{1 / 2}=\left\{\begin{array}{cc}
d, & t \ll t_{C M} \\
l_{M}^{2} / d, & t \gg t_{C M}
\end{array}\right.
$$

where $t_{C M}=M d^{2}$ is its spreading time and $l_{M}=\sqrt{t / M}$ is the quantum diffusion length. If the initial size $d$ of the atomic wave packet is approximately $10^{-6} \mathrm{~cm}$, we have $t_{C M} \sim 10^{-6}$ s for nucleus with the mass number $A \sim 100$. Hence, the width $D_{R}$ is of pure dispersion origin and grows linearly with time. The velocity of spreading is equal to $1 / M d$.

The time-dependent width of the RM wave packet $\left|\psi_{i}(\mathbf{r}, t)\right|^{2}$ is due to dispersive broadening of both the recoil and the massive neutrino. To estimate these effects, let us put $r \approx v_{i} t$ in Eq. (29). We get

$$
\Delta_{i}=\frac{1}{D_{i}}\left(l_{M}^{2}+\delta_{i}^{3} l_{m_{i}}^{2}\right)^{1 / 2} \approx \frac{l_{M}}{D_{i}}=\sqrt{\frac{t}{t_{i}}}
$$

where $t_{i}=M D_{i}^{2}$ is the spreading time caused only by a recoil particle. The contribution of a massive neutrino is negligible because of the relativistic suppression of the wave packet spreading. For $\Gamma \sim 1 \mathrm{~s}^{-1}$ and $A \sim 100$, the spreading time is $t_{i} \sim 10^{19} \mathrm{yr}$. This result is apparent for ultrarelativistic neutrinos, and we can put $\Delta_{i}=0$ in Eq. (28). In this limit, one finds the RM function

$$
\psi_{I^{\prime} M^{\prime}}(\mathbf{n}, r, t)=\frac{1}{\sqrt{2 \pi}} \xi_{I^{\prime} M^{\prime}}(\mathbf{n}) \sum_{i} R_{i}(r, t) U_{e i}^{*} c_{i \mathbf{k}_{i 0}}^{+}|0\rangle,
$$

where the radial function of the $i$ th massive neutrino is

$$
R_{i}(r, t)=\frac{\sqrt{2 \Gamma}}{r} \exp \left[i\left(k_{i 0} r-Q_{E C} t\right)-\frac{v_{i} t-r}{D_{i}}\right] \Theta\left(v_{i} t-r\right) .
$$

Here $\Theta$ is the unit step function. The function $R_{i}(r, t)$ is Lorentz covariant. The RM function (36) is normalized for ultra relativistic neutrinos by the condition

$$
\int d \mathbf{r} \sum_{M^{\prime}}\left|\psi_{I^{\prime} M^{\prime}}(\mathbf{n}, r, t)\right|^{2}=\sum_{i}\left|U_{e i}\right|^{2}=1 .
$$

The wave packet $\left|\psi_{I^{\prime} M^{\prime}}(\mathbf{r}, t)\right|^{2}$ is a superposition of three exponential wave packets of massive neutrinos with different sharp edges $r=v_{i} t$ and widths $D_{i}$. The later depends only on the dynamics of the decay process. The difference between the group velocities $v_{i}$ of these packets results in their separation. However, the separation is negligible compared with $D_{i}$ for times $t \ll 10^{12} \mathrm{yr}$. Therefore the wave packets corresponding to different mass eigenstates are in fact spatially inseparable. Hence, we can use an ultra relativistic approximation for $R_{i}$ with $D_{i}=D=1 / \Gamma$ and $v_{i}=1$. In this approximation the function (37) takes the form

$$
R_{i}(r, t)=R_{0}(r, t) \exp \left[i\left(k_{i 0} r-Q_{E C} t\right)\right]
$$

where

$$
R_{0}(r, t)=\frac{1}{r} \sqrt{\frac{2}{D}} \exp \left(-\frac{t-r}{D}\right) \Theta(t-r) .
$$

We now wish to consider the two-particle wave function, taken as the product of two functions (33) and (36), in the observable coordinates of the recoil nucleus and the neutrino. This normalized function has the form

$$
\Psi_{I^{\prime} M^{\prime}}=\Psi_{C M}\left((1-\alpha) \mathbf{r}_{n}+\alpha \mathbf{r}_{\nu}, t\right) \psi_{I^{\prime} M^{\prime}}\left(\mathbf{n},\left|\mathbf{r}_{\nu}-\mathbf{r}_{n}\right|, t\right) .
$$

The function does not factorize in these variables - a direct indication of the spatial entanglement of two particles. Each of the three massive neutrinos becomes entangled with the recoil nucleus because a neutrino is not emitted in a momentum eigenstate.

The joint recoil-neutrino wave packet $\sum_{M^{\prime}}\left|\Psi_{I^{\prime} M^{\prime}}\right|^{2}$ has an axially symmetrical shape with respect to the axis passing through the $\mathrm{CM}$ in the direction of the vector n. The packet increases with time in a transverse direction with velocity $1 / M d$ and in a longitudinal (along the axis) one with velocity $v_{n}+\left(1-v_{n}\right)=1$, where $v_{n}=v_{i} \epsilon_{i} / \epsilon_{n} \approx \alpha v_{i}$ is the velocity of a recoil. It is easy to see that the function $\Phi_{C M}$ takes its maximum value on the symmetry axis, along which the state is highly entangled. The probability density is proportional to a product of the Gaussian and exponential functions

$$
\begin{gathered}
\sum_{M^{\prime}}\left|\Psi_{I^{\prime} M^{\prime}}\right|^{2} \sim F_{I^{\prime}}(\theta) \exp \left\{-\frac{\left[(1-\alpha) r_{n}-\alpha r_{\nu}\right]^{2}}{D_{R}^{2}}\right\} \\
\quad \times \exp \left(-\frac{t-r_{\nu}-r_{n}}{D}\right) \Theta\left(t-r_{\nu}-r_{n}\right),
\end{gathered}
$$

where the angular modulation of the joint packet is determined by the function (angular distribution function)

$$
F_{I^{\prime}}(\theta)=\frac{1}{4 \pi}\left(1 \pm \frac{M_{F}}{I^{\prime}+1} \cos \theta\right) \quad \text { for } I^{\prime}=I \pm 1
$$


Modulation is due to the polarization of a parent ion. For a non-polarized ion we have $F_{I^{\prime}}=1 / 4 \pi$.

The Gaussian packet in Eq. (42) for fixed $r_{\nu}$ has the form

$$
\exp \left[\frac{(1-\alpha)^{2}}{D_{R}^{2}}\left(r_{n}-\frac{\alpha r_{\nu}}{1-\alpha}\right)^{2}\right],
$$

whereas for fixed $r_{n}$ it is equal

$$
\exp \left[\frac{\alpha^{2}}{D_{R}^{2}}\left(r_{\nu}-\frac{1-\alpha}{\alpha} r_{n}\right)^{2}\right] \text {. }
$$

Relative location of the peaks of these curves is determined by the condition

$$
(1-\alpha) r_{n}-\alpha r_{\nu}=0,
$$

which corresponds to the maximum of the wave packet (42). The $r_{\nu}$ dependent exponential and Gaussian curves overlap each other, if $r_{n}$ does not exceed its maximum value $v_{n} t$.

\section{EXPERIMENTAL IMPLEMENTATIONS}

Recent experiments concerned with atom-photon entanglement are dealing with generation and verification of an entangled pair. The special structure of the recoilneutrino wave packet offers new kinds of experiments. There are three types of possible experiments to detect a flavor neutrino and a recoil:

(i) Coincidence measurements, in which both the recoil and neutrino are detected. The flavor-changing process $e \rightarrow \beta$ is determined by the probability density

$$
\sum_{M^{\prime}} \mid\left\langle 0\left|\sum_{j} c_{j \mathbf{k}_{j 0}} U_{\beta j} \Psi_{I^{\prime} M^{\prime}}\left(\mathbf{r}_{n}, \mathbf{r}_{\nu}, t\right)\right|^{2} .\right.
$$

(ii) Noncoincidence measurements, when only neutrino is detected regardless of the recoil position. The flavor-changing process $e \rightarrow \beta$ is determined by the probability density

$$
\int d \mathbf{r}_{n} \sum_{M^{\prime}} \mid\left\langle 0\left|\sum_{j} c_{j \mathbf{k}_{j 0}} U_{\beta j} \Psi_{I^{\prime} M^{\prime}}\left(\mathbf{r}_{n}, \mathbf{r}_{\nu}, t\right)\right|^{2} .\right.
$$

(iii) Noncoincidence measurements, when only the recoil nucleus is detected regardless of the neutrino position. The probability density to detect recoil nucleus at the point $\mathbf{r}_{n}$ is

$$
\int d \mathbf{r}_{\nu} \sum_{M^{\prime}} \mid\left\langle 0\left|\sum_{j} c_{j \mathbf{k}_{j 0}} \Psi_{I^{\prime} M^{\prime}}\left(\mathbf{r}_{n}, \mathbf{r}_{\nu}, t\right)\right|^{2} .\right.
$$

The probability distributions (48) and (49) reveal no entanglement because all information about the position of one of the particles is lost completely.

Coincidence measurement. On the axis with highest entanglement, the probability density to detect recoil nucleus at the point $r_{n}$ together with the neutrino of flavor $\beta$ at the point $r_{\nu}$ is

$$
\begin{aligned}
& \frac{d P_{e \beta}}{d r_{n} d r_{\nu}}=F_{I^{\prime}}(\theta) \Psi_{C M}^{2}\left((1-\alpha) r_{n}+\alpha r_{\nu}, t\right) r_{n}^{2} r_{\nu}^{2} \\
& \times \sum_{i, j} U_{\beta i} U_{e i}^{*} U_{e j} U_{\beta j}^{*} R_{i}^{*}\left(r_{\nu}+r_{n}\right) R_{j}\left(r_{\nu}+r_{n}\right) .
\end{aligned}
$$

In ultrarelativistic approximation the second line is the probability of the $e \rightarrow \beta$ transition

$$
\sum_{i}\left|U_{e i}\right|^{2}\left|U_{\beta i}\right|^{2}+2 \sum_{i>j}\left|U_{\beta i} U_{e i}^{*} U_{e j} U_{\beta j}^{*}\right| \cos \left(\frac{\Delta m_{i j}^{2}}{2 Q_{E C}} r+\gamma\right) \text {, }
$$

where $\Delta m_{i j}^{2}=m_{i}^{2}-m_{j}^{2}$ and $\gamma=\arg \left(U_{\beta i} U_{e i}^{*} U_{e j} U_{\beta j}^{)}\right)$.

We choose the direction of the axis corresponding to the maximum value of $F_{I^{\prime}}(\theta)$, which will be denoted by $F_{I^{\prime}}$. The neutrino detector $\mathrm{N}$, which fixes the flavor of neutrino states by some charged current process, and the recoil one $\mathrm{R}$ to register the nucleus are connected with a coincidence circuit and located on this axis on both sides of a parent ion confining volume (that is approximately the CM position) at distances from it, respectively, $L_{\nu}$ and $L_{n}$. If $L_{\nu}$ and $L_{n}$ satisfy Eq. (46), the amplitude of oscillations will be equal

$$
\frac{2 F_{I^{\prime}} L_{n}^{2}}{\pi^{3 / 2} D D_{R}^{3}(t)} \exp \left[-2 \Gamma\left(t-L_{\nu}-L_{n}\right)\right]
$$

The amplitude conforms to highest spatial entanglement and can serve as its verification. The detection time $t$ depends on the lifetime of a parent ion. For short lifetimes, the length of the joint wave packet may be equal to distance between the detectors, that is $t=L_{\nu}+L_{n}$. In this case, the amplitude (52) will be maximum. This condition is impossible for parent ions in a Penning trap because their lifetime must exceed the time it takes to prepare them in the trap. The latter is of the order of seconds. In such a case, the measurement time $t>1 / \Gamma$ should be taken so as to minimize the exponent in Eq. (52).

It follows from Eq. (51) that the expression for the probability (50) contains the well-known oscillation phase

$$
\phi_{i j}=\frac{\Delta m_{i j}^{2}}{2 Q_{E C}}\left(L_{\nu}+L_{n}\right) .
$$

For two neutrino mixing, i.e. in the case of $e \rightarrow \mu$ (i.e. $\beta=e, \mu)$ oscillations, the mixing matrix is

$$
U=\left(\begin{array}{cc}
\cos \eta & \sin \eta \\
-\sin \eta & \cos \eta
\end{array}\right)
$$


where $\eta$ is the mixing angle. Then, one finds from Eq. (51) the probabilities

$$
\mathcal{W}_{e e}=1-\sin ^{2}(2 \eta) \sin ^{2} \phi_{12}, \quad \mathcal{W}_{e \mu}=\sin ^{2}(2 \eta) \sin ^{2} \phi_{12},
$$

where $\phi_{12}=\pi\left(L_{\nu}+L_{n}\right) / L_{0}$ and $L_{0}=4 \pi Q_{E C} / \Delta m^{2}$ $\left(\Delta m^{2}=m_{2}^{2}-m_{1}^{2}>0\right)$ is the oscillation length. The final expression for the coincidence probability density is

$$
\begin{gathered}
\frac{d P_{e \beta}}{d r_{n} d \Omega_{n} d r_{\nu} d \Omega_{\nu}}=\frac{2 F_{I^{\prime}} L_{n}^{2}}{\pi^{3 / 2} D D_{R}^{3}(t)} \\
\times \exp \left[-2 \Gamma\left(t-L_{\nu}-L_{n}\right)\right] \mathcal{W}_{e \beta} \mathcal{W}_{n},
\end{gathered}
$$

where $\mathcal{W}_{n}=1$ is the probability of a recoil registration. For the detectors connected to coincidence circuit with one output (the "and circuit"), a signal disappearance at certain positions of the detectors $\mathrm{N}$ and $\mathrm{R}$ is caused by oscillating probability $\mathcal{W}_{e \mu}$ in the muon neutrino channel. The recoil nucleus does not oscillate. To observe the spatial correlation and neutrino oscillations, one needs the correlation experiment, in which an experimental event involves the registration of a neutrino simultaneously with a recoil nucleus in two detectors. One can fix the distance from source to one of the detectors and change the distance to another one. Here, too, both neutrino oscillations and a recoil may be observed, but with a far lower amplitude due to the Gaussian factor of Eq. (42).

Let us examine the conditions for the observation of oscillation patterns. The first condition is trivial: the source-detector distance $L_{\nu}$ should be of the order of, or greater than, the oscillation lengths $L_{\text {osc }}$. The second condition is the coherence of different mass eigenstates, necessary for the neutrino and recoil oscillations to be observed. Coherence is preserved over distances not exceeding the coherence length $L_{c o h}$. The latter is defined as the distance at which the phase difference due to energy spreading obeys the equation

$$
\phi_{i j}\left(Q_{E C}\right)-\phi_{i j}\left(Q_{E C}+\Gamma\right)=2 \pi .
$$

We find $L_{c o h}=L_{o s c} Q_{E C} / \Gamma \sim 10^{21} L_{o s c}$. Such a large correlation length arises because the emitter is in a pure quantum state that is described by a state vector (9).

Neutrino detection. This is a well-established experiment for studying neutrino oscillation. Integration over $d \mathbf{r}_{n}$ in Eq. (48) can be done approximately since the width of the Gaussian packet (44) is much smaller than that of the exponential, if $\Gamma t \ll 10^{10}$. Indeed, we have

$$
\frac{D_{R}}{(1-\alpha) D} \approx \frac{1}{D} \sqrt{d^{2}+\frac{t^{2}}{M^{2} d^{2}}} \approx \frac{\Gamma t}{2 M d} \sim 10^{-10} \Gamma t
$$

The integral is calculated as follows

$$
\begin{gathered}
\int \Psi_{C M}^{2}\left((1-\alpha) \mathbf{r}_{n}+\alpha \mathbf{r}_{\nu}\right) R_{i}^{*}\left(\left|\mathbf{r}_{\nu}-\mathbf{r}_{n}\right|\right) R_{j}\left(\left|\mathbf{r}_{\nu}-\mathbf{r}_{n}\right|\right) d \mathbf{r}_{n} \\
\approx R_{i}^{*}\left(\frac{r_{\nu}}{1-\alpha}\right) R_{j}\left(\frac{r_{\nu}}{1-\alpha}\right) \int \Psi_{C M}^{2}\left((1-\alpha) \mathbf{r}_{n}\right) d \mathbf{r}_{n} .
\end{gathered}
$$

Let the position of a neutrino detector be given by the distance $L_{\nu}$ from the emitter, then the probability density to detect the neutrino of flavor $\beta$ at this distance is

$$
\begin{aligned}
& \frac{d P_{e \beta}}{d r_{\nu} d \Omega_{\nu}}=\frac{2 F_{I^{\prime}}}{D} \exp \left[-2 \Gamma\left(t-L_{\nu}\right)\right]\left\{\sum_{i}\left|U_{e i}\right|^{2}\left|U_{\beta i}\right|^{2}\right. \\
& \left.\quad+2 \sum_{i>j}\left|U_{\beta i} U_{e i}^{*} U_{e j} U_{\beta j}^{*}\right| \cos \left(2 \pi \frac{L_{\nu}}{L_{i j}^{\nu}}+\gamma\right)\right\} .
\end{aligned}
$$

Recoil nucleus detection. Integral (49) is calculated in perfect analogy to (48) if $\Gamma t \ll 10^{5}$ As a result, we obtain the following expression for the probability density to detect recoil at a distance $L_{n}=v_{n} t$ from the parent ion

$$
\frac{d P_{n}}{d r_{n} d \Omega_{n}}=\frac{F_{I^{\prime}} \Gamma}{v_{n}}
$$

The joint wave function of the recoil-neutrino pair allows to calculate probability densities of the considered processes. For full description of oscillation experiments, it is necessary to take into account a detection process. However, this is different problem.

\section{CONCLUSION}

In summary, we have found an accurate analytical solution for the joint quantum state of an electron neutrino and a recoil nucleus following the electron capture decay of a hydrogenlike ion. The evolution of the state provides an exactly calculable illustration of the famous EinsteinPodolsky-Rosen thought experiment. The new effect is the entanglement between the recoil and the coherent superposition of the three massive neutrinos. Such a superposition cannot be observed in the experiments involving discrete states of an atom-photon pair because the polarization of an emitted photon is inevitably entangled with the spin state of an atom.

We have shown that each of the three massive neutrinos is not emitted in a momentum eigenstate, and so the recoil becomes entangled with the superposition of three neutrinos. However, the total energy is conserved in the course of EC decay, and the decay energy converts to the kinetic energy of the reoil-neutrino pair. Thus, we have rigorously proved that the neutrino mass eigenstates composing the electron eigenstate produced in EC decay have the same energy. It should be emphasized that although a particular type of decay has been treated, the results of our calculations are applicable to other twobody weak decays due to the common features of the initial-value problem for noninteracting particles.

The most peculiar aspect of our solution is the twoparticle system in a pure quantum state, where the particles, a recoil and a massive neutrino, are mixed with each other. Such an entanglement opens up an opportunity for correlative experiments and the investigation of environment-induced decoherence. This is the main 
distinction of our approach from the field-theoretical one [11], where a neutrino enters as unobserved intermediate state. We have suggested the correlative experiment which allows to observe neutrino oscillations and a recoil simultaneously. However, a considerable progress in the detection methods of neutrinos and recoil nuclei is necessary to carry out such measurements with a single ion.

\section{Acknowledgments}

The author is grateful to A. L. Barabanov and Y. Litvinov for critical comments. This research was supported by Grant NS-7235-2010.2 from the Russian Ministry of Education and Science.

\section{Appendix}

We consider the integral

$$
\mathcal{I}=\int_{0}^{\infty} \frac{k_{i}(\epsilon)}{v_{i}(\epsilon)} \frac{\exp \left\{i k_{i}(\epsilon) r-i\left[\epsilon+\frac{k_{i}^{2}(\epsilon)}{2 M}-\frac{\mathbf{p k}_{i}(\epsilon)}{M}\right] t\right\}}{\epsilon-Q_{E C}+\frac{k_{i}^{2}(\epsilon)}{2 M}-\frac{\mathbf{p k}_{i}(\epsilon)}{M}+i \Gamma} d \epsilon
$$

where the function $k_{i}(\epsilon)$ is given by power series (26). In the lowest orders of the small parameters $\alpha$ and for $\Gamma \ll Q_{E C}$ the pole of the integrand is

$$
\epsilon_{p}=Q_{E C}-i \Gamma+\left(\frac{\mathbf{p k}_{i 0}}{M}-\frac{k_{i 0}^{2}}{2 M}\right)\left(1+\frac{\mathbf{p n}}{M v_{i}}-\frac{k_{i 0}}{M v_{i}}\right) .
$$

We may extend the lower limit in (A.1) to $-\infty$, since the contribution to the integral falls of sharply with increasing $\left|\epsilon_{i}\right|$ owing to $\Gamma \ll Q_{E C}$. The integral involves the exponential factor $\exp \left[-i q_{i}\left(\epsilon-Q_{E C}\right)^{2}\right]$ with

$$
\begin{gathered}
q_{i}=\frac{1}{2}\left[\frac{t}{M v_{i}^{2}}+\frac{\delta_{i}^{3}}{m_{i} v_{i}^{3}}\left(r+\frac{\mathbf{p n}}{M} t-\frac{k_{i 0}}{M} t\right)\right] \\
\approx \frac{1}{2}\left(\frac{t}{M v_{i}^{2}}+\frac{\delta_{i}^{3} r}{m_{i} v_{i}^{3}}\right),
\end{gathered}
$$

which prevents the use of the residue method. To overcome the problem, we have to use the Fourier transformation of this factor [15]

$$
\exp \left[-i q \varepsilon^{2}\right]=\frac{1}{\sqrt{4 i \pi q}} \int_{-\infty}^{\infty} \exp \left(\frac{i x^{2}}{4 q}+i \varepsilon x\right) d x .
$$

Then, we first evaluate the integral

$$
\oint_{C} \frac{k_{i}(\epsilon) \exp \left[i \mathcal{P}(\epsilon)+i\left(\epsilon-Q_{E C}\right) x\right]}{v_{i}(\epsilon)\left(\epsilon-\epsilon_{p}\right)} d \epsilon,
$$

where the contour $C$ encloses the pole (A.2), and the exponent $\mathcal{P}$ is

$$
\begin{gathered}
\mathcal{P}(\epsilon)=k_{i 0} r-\left(Q_{E C}+\frac{k_{i 0}^{2}}{2 M}-\frac{\mathbf{p k}_{i 0}}{M}\right) t \\
+\left[\frac{r}{v_{i}}-\left(1-\frac{\mathbf{p n}}{M v_{i}}+\frac{k_{i 0}}{M v_{i}}\right) t\right]\left(\epsilon-Q_{E C}\right) .
\end{gathered}
$$

The integral is easily calculated, and Eq. (A.1) takes the form

$$
\mathcal{I}=-\frac{2 i \pi k_{i 0} e^{i \mathcal{P}}}{\sqrt{4 i \pi q_{i}} v_{i}} \int_{r / v_{i}-t}^{\infty} \exp \left[\frac{i x^{2}}{4 q_{i}}+i x\left(\epsilon_{p}-Q_{E C}\right)\right] d x,
$$

where for $\Gamma \ll Q_{E C}$ the expression for the exponent taken in the pole has the form

$$
\begin{gathered}
\mathcal{P}=\mathcal{P}\left(\epsilon_{p}\right)=k_{i 0} r\left(1-\frac{\alpha}{2}+\frac{\alpha^{2}}{2}\right)-Q_{E C}\left(1+\frac{\alpha^{3}}{2}-\frac{\alpha m_{i}^{2}}{2 M^{2}}\right) t \\
+\alpha \mathbf{p r}\left(1-\frac{3 \alpha}{2}+2 \alpha^{2} \frac{v_{i} t}{r}\right)+i \Gamma\left[\left(1-\alpha^{2}\right) t-\frac{r}{v_{i}}(1-\alpha)\right] \\
+\alpha \frac{(\mathbf{p n})^{2}}{M}\left(\frac{r}{v_{i}}-\frac{5 \alpha}{2} t\right)+\alpha \frac{(\mathbf{p n})^{3}}{M^{2} v_{i}} t .
\end{gathered}
$$

We have approximately

$$
\mathcal{P} \approx k_{i 0} r+\alpha \mathbf{p r}-Q_{E C} t+i \Gamma\left(t-r / v_{i}\right) .
$$

The last two terms in the expression A.8 can be dropped because they are small compared to the term proportional to $\mathbf{p}^{2}$ in the exponent of Eq. (22). It is seen from Eq. (A.8) that the energy of a pair of $i$ th neutrino and a recoil is the same for all pairs with great accuracy and is equal to the decay energy $Q_{E C}$. Small deviations from $Q_{E C}$ are attributable to the approximate calculation of the pole (A.2): the more precisely it is defined, the less are these deviations.

Finally, the integral (A.7) can be transformed into the error function

$$
\mathcal{I}=-\frac{i \pi k_{i 0}}{v_{i}}\left(1-\operatorname{Erf} \frac{r / v_{i}-t+i q_{i} \Gamma}{\sqrt{4 i q_{i}}}\right) e^{i \mathcal{P}} .
$$

(2010).

[4] B. Kayser, J. Kopp, R. G. H. Robertson, and P. Vogel, Phys. Rev. D 82, 093003 (2010).

[5] S. Nussinov, Phys. Lett. B 63, 201 (1976).

[6] B. Kayser, Phys. Rev. D 24, 110 (1981).
(1978).

[3] K. Nakamura and S. T. Petcov, J. Phys. G 37, 075021 
[7] C. Giunti, C. W. Kim, and U. W. Lee, Phys. Rev. D 44, 3635 (1991).

[8] A. D. Dolgov, L. B. Okun, M. V. Rotaev, and M. G. Shchepkin, arXiv:0407189v2.

[9] C. Giunti and C. W. Kim, Phys. Rev. D 58, 017301 (1998).

[10] W. Grimus and P. Stockinger, Phys. Rev. D 54, 3414 (1996).

[11] W. Grimus, P. Stockinger, and S. Mohanty, Phys. Rev. D 59, 013011 (1998).

[12] Jun Wu, J. A. Hutasoit, D. Boyanovsky, and R. Holman, Phys. Rev. D 82, 013006 (2010).

[13] E. K. Akhmedov and A. Yu. Smirnov, Phys. Atom. Nucl. 72, 1363 (2009).

[14] Attempts to follow these basic principles have been made in the works of M. Nauenberg, Phys. Lett. B 447, 23 (1999), and B. Meszéna and A. Patkós, Mod. Phys. Lett. A 26, 101 (2011).

[15] M. V. Fedorov, M. A. Efremov, A. E. Kazakov, K. W. Chan, C. K. Law, and J. H. Eberly, Phys. Rev. A 69, 052117 (2004).

[16] M. V. Fedorov, M. A. Efremov, A. E. Kazakov, K. W. Chan, C. K. Law, and J. H. Eberly, Phys. Rev. A 72,
032110 (2005).

[17] A. Einstein, B. Podolsky and N. Rosen, Phys. Rev. 47, 777 (1935).

[18] J. T. Goldman, Mod. Phys. Lett. A 25, 479 (2010).

[19] C. Kurtsiefer, O. Dross, D. Voigt, C. R. Ekstrom, T. Pfau, and J. Mlynek, Phys. Rev. A 55, R2539 (1997).

[20] A. D. Dolgov, A. Yu. Morozow, L. B. Okun, and M. G. Shchepkin, Nucl. Phys. B 502, 3 (1997).

[21] V. Weisskopf and E. Wigner, Z. Phys. 63, 54 (1930).

[22] Y. Litvinov et al., Phys. Lett. B 664, 162 (2008).

[23] Y. Litvinov and F. Bosch, private communication (2011).

[24] The volume of a normalized box will be taken 1 throughout this paper.

[25] C. Giunti, C. W. Kim, and U. W. Lee, Phys. Rev. D 45, 2414 (1992).

[26] D. A. Varshalovich, V. K. Khersonsky and A. N. Moskalev, Quantum Theory of Angular Momentum (World Scientific, Singapore, 1988).

[27] C. K. Law and J. H. Eberly, Phys. Rev. Lett 92, 127903 (2004).

[28] L. D. Landau and E. M. Lifshitz, The Classical Theory of Fields (London, Pergamon, 1962). 